\title{
Colistin resistance in Acinetobacter baumannii isolated from critically ill patients: clinical characteristics, antimicrobial susceptibility and outcome
}

\author{
Bukhari Elham ${ }^{1}$, Alotaibi Fawzia ${ }^{2}$
}

1. Department of Pediatrics, College of Medicine, King Saud University, Riyadh 11442, Saudi Arabia.

2. Department of Pathology and Laboratory Medicine, College of Medicine, King Saud University and King Saud University Medical City, Riyadh, Saudi Arabia.

\begin{abstract}
:
Background: Acinetobacter baumannii (AB) is increasingly becoming a clinically relevant organism due to the rising number of associated nosocomial infections. The therapeutic options are extremely minimal because of its ability to develop resistance to all available antimicrobials, including colistin (CST). Data on the clinical and microbiological characteristics of colistin-resistant A. baumannii infections remain scarce to date.

Methods: In this prospective study, clinical isolates of colistin resistance among Acinetobacter strain was evaluated from the database of Microbiology Laboratory of King Khalid University Hospital, Saudi Arabia.

Results: In a total of 142 patients with 136 Acinetobacter isolates, Acintobacter baumannii was the predominant serotype 73\% of the isolates and Acinetobacter lwoffii constituted $27 \%$ of the isolate. There was $8.5 \%$ colistin resistant isolates with colistin E-test MIC $>4$. The clinical characteristics were determined for colistin resistant Acinetobacter baumannii. All patients were critically ill and $64 \%$ of them were hositalized in the Intensive Care Unit (ICU). All patients have been previously given antibiotics. Other associated clinical characteristics included; morbid obesity and sleeve gastrectomy (21\%), mechanical ventilation and central venous catheter (50\%). High mortality rate was found(28\%).

Conclusion: There is an increase of colistin resistance among clinical isolates of Acinetobacter baumannii causing serious infections especially in critically ill patients.
\end{abstract}

Keywords: Acinetobacter baumannii, colistin resistance, clinical characteristics.

DOI: https://dx.doi.org/10.4314/ahs.v19i3.13

Cite as: Elham B, Faw zia A. Colistin resistance in Acinetobacter baumannii isolated from critically ill patients: clinical characteristics, antimicrobial susceptibility and outcome. Afri Health Sci. 2019;19(3): 2400-2406. bttps:// dx.doi.org/10.4314/abs.v19i3.13

\section{Introduction}

Acinetobacter baumannii (AB) is one of the important multidrug resistant Gram-Negative nosocomial pathogens. It is responsible for many serious infections such as pneumonia, sepsis, urinary tract and wound infections. ${ }^{1}$ Multiple factors lead to colonization with Acinetobacter baumannii, such as prolonged hospital stay, post- surgical procedures, ICUadmission and prior treatment with broad spectrum antibiotics. ${ }^{2,3}$ Recently, Acinetobacter strains exhibited elevated rates of carbapenem resistance (as high as 58\%). ${ }^{4}$ Colistin, a natural substance produced by Bacillus polymyxa and a cationic lipopeptide (cyclic decapeptide) was discovered in 1949. Colistin was considered as one of the

\section{Corresponding author:}

Bukhari Elham,

Department of Pediatrics, College of Medicine, King Saud University, Riyadh 11442, Saudi Arabia.

Email: ebukhari@ksu.edu.sa last therapeutic options for treatment of the multiresistant Acinetobacter baumannii infection. ${ }^{5}$ It has been recommended for the treatment of ventilator associated pneumonia caused by drug resistant gram negative organism. ${ }^{6}$ Recently colistin has been used as rescue therapy for severe infection. ${ }^{7}$ However, resistance to colistin has recently been described worldwide. ${ }^{8-12}$ Few data on Acinetobacter spp. infection and colistin resistance in Saudi Arabia is currently found in the literature. ${ }^{13,14}$ The objectives of the present study were to evaluate the clinical characteristics, antimicrobial susceptibility testing and outcomes of patients infected with colistin-resistant Acinetobacter baumannii.

\section{Methods}

\section{Study design}

This study was a prospective review conducted in a teaching hospital located in Riyadh, Saudi Arabia using the Microbiology Laboratory database and the daily patients' records from February 2015 through April 2016.
African
Health Sciences

(C) 2019 Elham et al. Licensee African Health Sciences. This is an Open Access article distributed under the terms of the Creative commons Attribution License (https://creativecommons.org/licenses/BY/4.0), which permits unrestricted use, distribution, and reproduction in any medium, provided the original work is properly cited.

African Health Sciences Vol 19 Issue 3, September, 2019 


\section{Bacterial isolates}

A total of 136 non-duplicate clinical isolates of Acinetobacter isolates obtained from 142 patients, of which 14 patients had multiple infections with different Acinetobacter species. Various specimens were collected from different sites including blood, genitourinary tract, respiratory tract and wounds specimens. Multidrug resistant bacteria are described as being resistant to at least one agent in three or more antibiotic categories.

\section{Antimicrobial susceptibility testing}

The identification of the isolates and colistin susceptibility testing were performed by Vitek 2 (bioMérieux, France), and the colistin-resistant MICs were confirmed by E test methods, according to CLSI recommendations. ${ }^{15}$ The $\mathrm{E}$ test method was previously validated on the microbiology laboratory using broth microdilution (Trek Diagnostics Systems), according to CLSI recommendations. ${ }^{15}$ The susceptibility categories were interpreted according to break-points of CLSI guidelines. ${ }^{16}$ for non-fermentative isolates and EUCAST. ${ }^{17}$ for Enterobacteriaceae mem- bers. Colistin minimum inhibitory concentrations (MICs) $>2 \mu \mathrm{g} / \mathrm{mL}$ were considered resistant. ${ }^{18}$

\section{Patient's clinical data}

The clinical characteristics associated with acquiring multiresistant Acinetobacter baumannii were determined by reviewing the clinical data recorded in the patient's medical chart.

\section{Results \\ Characteristics of isolates}

Of the 227 Acinetobacter isolates obtained from (142) patient, 64 duplicate isolates were excluded. Total of 163 Acinetobacter isolates were included and 10\% (14/142) had more than one site of infection. Almost 41.8\% (68/163) of the Acinetobacter isolates were multidrug resistant. Over the study period, the prevalence of monthly reported Acinetobacter spp. was recorded in the data base of the Microbiology laboratory and it was steadily increasing reaching the highest prevalence $(30 \%)$ of the total 136 Acinetobacter isolates which was observed in March (2016) [Figure 1].

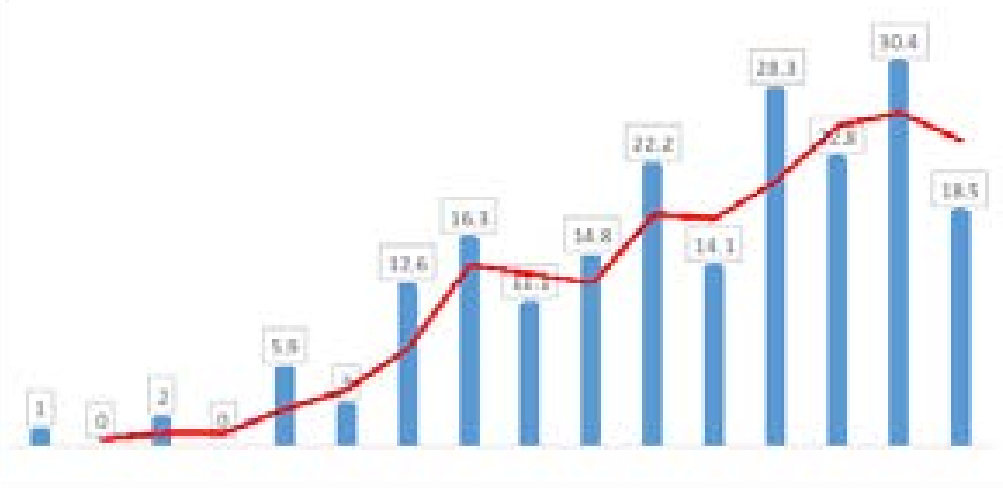

Figure 1: Prevalence of 136 Acinetobacter species isolates over the study period

Acinetobacter baumannii isolates 119 (73\%) were the most common identified isolates of all Acinetobacter species. Fourteen Acinetobacter baumannii isolates (14/163, (8.6\%) were colistin resistant, (MIC: $\geq 4 \mathrm{mg} / \mathrm{ml}$ ).

\section{Site of infection}

The most common sites of specimens included respira- tory tract $(6 / 14 ; 42 \%)$, urine $(2 / 14 ; 14 \%)$, wound $(1 / 14$; $7 \%)$, blood $(4 / 14 ; 28 \%)$, and tissue $(1 / 14 ; 7 \%)$.

\section{Clinical and demographics of patients}

Demographic data and associated clinical characteristics and comorbid diseases that were more prevalent in the 14 patients infected with colistin resistant Acinetobacter baumannii are illustrated in [Table 1]. 
Table 1. Clinical characteristics of patients infected with colistin resistant Acinetobacter baumannii

\begin{tabular}{|l|c|c|}
\hline Clinical characteristics & $\mathbf{n}=\mathbf{1 4}$ & $\mathbf{\%}$ \\
\hline Age (mean) & 12yr-91yr. & \\
\hline Gender & & \\
\hline - Male & 9 & 63 \\
\hline - Female & 5 & 41 \\
\hline Admission & & \\
\hline - ICU & 9 & 64 \\
\hline - Medical & 6 & 43 \\
\hline - Surgical & 3 & 21 \\
\hline Medical/Surgical Comorbidity: & & \\
\hline - Diabetes mellitus & 4 & 28 \\
\hline - Hypertension & 4 & 28 \\
\hline - Morbid Obesity & 3 & 21 \\
\hline - Chronic renal failure & 3 & 21 \\
\hline - Heart disease & 4 & 28 \\
\hline - Malignancy & 2 & 14 \\
\hline - Immunosuppression & 1 & 7 \\
\hline - Septic shock & 2 & 14 \\
\hline - Surgery & 5 & 35 \\
\hline - Sleeve Gastrectomy & 3 & 21 \\
\hline Hospitalization (last 3 months) & 7 & 50 \\
\hline Invasive devices & & \\
\hline - Mechanical ventilation & 7 & 50 \\
\hline - Central venous catheter & 7 & 50 \\
\hline - Urinary catheter & 5 & 35 \\
\hline Death & 4 & 28 \\
\hline ICU: Intensive Cre Unit & & \\
\hline
\end{tabular}

ICU; Intensive Care Unit

Of the 14 patients between the age of (12-91), there were 9 male $(64 \%)$ and 5 females $(35 \%)$ and one pediatric patient $(7 \%)$ who had lymphoma. Nine patients were admitted in the intensive care unit (64\%). Four (28\%) patients had diabetes mellitus and hypertension. chronic renal failure, morbid obesity, and sleeve gastrectomy were identified in 3 patients $(21 \%)$ respectively. Sleeve gastrectomy was done one year, seven months and three months prior to the study in three patients. Seven $(50 \%)$ patients were mechanically ventilated. Central line and urinary catheter were used in $(50 \%, 43 \%)$ respectively. Prior hospitalization was documented in all $A$. baumannii colistin resistant infected patients $(100 \%)$. All patients received antibiotics before the diagnosis of $\mathrm{AB}$ infection including carbapenem $(78.5 \%)$ colistin $(28 \%)$, vancomycin $(64 \%)$, tazocin (50\%), linezolid (14\%), aminoglycoside (14\%) and antifungal agents (43\%). High mortality rate $(28 \%)$ was found among patients with $\mathrm{AB}$ colistin resistant infection in spite of intensive treatment. Treatment of patients included colistin in combination with aminoglycosides or carbapenem. Data obtained after analyzing resistance to $\mathrm{AB}$ strains isolated showed that this bacteria's antibiotic resistance was high. The Minimum Inhibitory Concentration (MIC) of colistin against the various Acinetobacter baumannii for which the MIC E-test was performed is presented in [Table 2]. 
Table 2. Previous antibiotics used for the last 3 months in patients with Acinetobacter baumannii infection

\begin{tabular}{|l|l|c|}
\hline Antibiotic & $\mathrm{N}$ & $\%$ \\
\hline - Total & 14 & 100 \\
\hline - Colistin & 5 & 35 \\
\hline - Carbapenem & 10 & 71 \\
\hline - Glycopeptide (vancomycin) & 9 & 64 \\
\hline - Cephalosporin & 3 & 21 \\
\hline - Aminoglycoside & 2 & 14 \\
\hline - Quinolone & 0 & 0 \\
\hline - Piperacillin-tazobactam & 7 & 50 \\
\hline - Linezolide/daptomycine & 2 & 14 \\
\hline - Tigecycline & 1 & 7 \\
\hline - Metronidazole & 2 & 16 \\
\hline - Antifungal & 6 & 43 \\
\hline
\end{tabular}

\section{Discussion}

In this study the prevalence of Acinetobacter isolates was found to be steadily increasing. This finding was reported by other studies; a study from Iran showed that, the prevalence of drug resistance among the clinical samples of A.baumannii isolated against most of the antibiotics is very high. ${ }^{19}$ Another study from Romania has shown that a large number of $A$. baumannii strains were resistant to the tested antibiotics. The lowest resistance was recorded for ampicillin and sulbactam and the highest resistance rate was recorded for ceftazidime and imipenem. ${ }^{20-24}$

Acinetobacter baumannii isolates were the most common identified isolates of all Acinetobacter species, (73\%) this finding was similar to other studies. ${ }^{25,26}$

In this study a large number of colistin-resistant/carbapenem-resistant $A$. baumannii was observed. Similar to our finding, the emergence of colistin resistant $A$. baumannii has been observed in several countries. ${ }^{27-30}$

A study from Central Greece showed a significant increase of colistin-resistant/carbapenem-resistant $A$. baumanniias a total of 86 colistin-resistant/carbapenem-resistant out of 1228 A. baumannii were collected between 2012 and $2014 .{ }^{31}$

The age of the patients was variable between 4-90 years; all of them were critically ill and had chronic disease such as hypertension, chronic renal failue, morbid obesity and malignancy. All of them had prior hospital admission and were treated with different antibiotics including carbapenem, aminoglycoside and vancomycin. More than 50\% of the patients required central line and urinary catheters.

Knowledge of these possible risk factors for Colistin-resistant $A$. baumannii is a challenge for antibiotic treatment and infection control policies. We found that the majority of the patients were admitted in the intensive care unit $(64 \%)$. Mechanical ventilation in $(50 \%)$, Prior hospital admission and antibiotic use were documented in all patients $(100 \%)$. Similar findings were reported by Qureshi et al; in his study all patients but one were in the ICU at the time of isolation of colistin- resistant $A$. baumannii and $(65 \%)$ of the patient used ventilator. ${ }^{32}$

In this study, among the antibiotics that had been used previously carbapenem was used in $(78.5 \%)$ of the patients, while colistin was used in $(28 \%)$. In Qureshi et al study, $95 \%$ of the patients had received intravenous and/ or inhaled colistin for the treatment of carbapenem-resistant, colistin-susceptible $A$. baumannii infection prior to identification of colistin-resistant isolates. ${ }^{32}$

Another study from Turkey reported that previous quinolone use in the last three months $(\mathrm{P}=.003$; $\mathrm{RR}: 3.2$; 95\% CI:1.5-6,7) and previous colistin use in the last three months (P=.001; RR: 3.6; 95\% CI: 1.63-7.99) were significant risk factors in the multivariate analysis associated with infection by colistin-resistant microorganisms. ${ }^{33}$ 
In spite of intensive treatment, $28 \%$ of our patients died by 30 days. A study from USA reported high mortality rates among almost all patients with colistin resistance $\mathrm{AB}$ and suffering from ventilator-associated pneumoniae. ${ }^{32}$ Another study from Brazil reported 7 out of 20 isolates were colistin resistant and 8 patients died during therapy. ${ }^{34}$

A retrospective cohort study was conducted in the medical/surgical ICU at Zhongshan Hospital in Shanghai, China. Adult patients meeting the criteria of A.baumannii VAP from January 2012 to October 2015 were enrolled. Seventy-eight patients were included in this study. The 30 -day mortality rate in ICU for the patients was $37.2 \%{ }^{35}$ We believe that this is a high mortality rate in comprasion to our study where the mortality rate was $(28 \%)$.

To our knowledge, our study involved 14 unique patients with infection due to colistin-resistant $\mathrm{AB}$.

The most important limitation of this study was that it was of a small number of samples, probably because it was a prospective study that was carried out in relatively short time. Despite all limitations, this study represents the largest series describing clinical characteristics of colistin-resistant AB. Our data highlights an emerging clinical problem. Hence, early detection and optimization of infection control practices are required.

\section{Acknowledgment}

This project was financially supported by King Saud University through Vice Deanship of Research chairs.

\section{Conflict of interest}

None declared.

\section{References}

1. Sievert DM, Ricks P, Edwards JR, et al. Antimicrobial-resistant pathogens associated with healthcare-associated infections: summary of data reported to the National Healthcare Safety Network at the Centers for Disease Control and Prevention, 2009-2010. Infect Control Hosp Epidemiol. 2013; 34:1-14.

2. Joel Fishbain1 and Anton Y. Peleg. Treatment of Acinetobacter Infections Clinical Infectious Diseases. 2010; 51(1):79-84 3. 1. Maragakis LL, Perl TM. Acinetobacter baumannii: epidemiology, antimicrobial resistance, and treatment options. Clin Infect Dis. 2008; 46(8):1254-1263.

4. Rhomberg PR, Jones RN. Summary trends for the Meropenem Yearl Susceptibility Test Information Collec- tion Program: a 10-year experience in the United States (1999-2008). Diagn Microbiol Infect Dis. 2009; 65:414-26. PubMed

5. Zubair A. Qureshi,1 Lauren E. Hittle,2 Jessica A. O'Hara,1 Jesabel I. Rivera,1 Alveena Syed,1 Ryan K. Shields, Anthony W. Pasculle,3 Robert K. Ernst,2 and Yohei Doi. Colistin-Resistant Acinetobacter baumannii: Beyond Carbapenem Resistance. Clinical Infectious Diseases. 2015;60(9):1295-303

6. American Thoracic Society; Infectious Diseases Society of America. Guidelines for the management of adults with hospital-acquired (2005) ventilator-associated, and healthcare-associated pneumonia. Am J Respir Crit Care Med. 171: 388-416.

7. Oleksiuk LM, Nguyen MH, Press EG, Updike CL, O'Hara JA, et al. (2014). In vitro responses of Acinetobacter baumannii to two- and three-drug combinations following exposure to colistin and doripenem. Antimicrob Agents Chemother. 58: 1195-1199.

8. Yau W, Owen RJ, Poudyal A, et al. Colistin hetero-resistance in multi-drug-resistant Acinetobacter baumanniiclinical isolatesfrom the Western Pacific region in the SENTRY antimicrobialsurveillance programme. J Infect. 2009;58:138-44

9. Gales, A. C., R. N. Jones, and H. S. Sader. 2006. Global assessment of the antimicrobial activity of polymyxin B against 54731 clinical isolates of Gramnegative bacilli: report from the SENTRY Antimicrobial Surveillance Programme (2001-2004). Clin. Microbiol. Infect. 12:315-321.

10. Hernan, R. C., B. Karina, G. Gabriela, N. Marcela, V. Carlos, and F. Angela.2009. Selection of colistin-resistant Acinetobacter baumannii isolates in post neurosurgical meningitis in an intensive care unit with high presence of hetero resistance to colistin. Diagn. Microbiol. Infect. Dis. 65:188- 191.

11. Park, Y. K., J. Y. Choi, S. I. Jung, K.span style="font-family:'Times New Roman'; letter-spacing:0.7pt"> H. Park, H. Lee, D. S. Jung, S. T. Heo, S. W. Kim, H. H. Chang, H. S. Cheong, D. R. Chung, K. R. Peck, J. H. Song, and K. S. Ko. 2009. Two distinct clones of carbapenem-resistant Acinetobacter baumannii isolates from Korean hospitals. Diagn. Microbiol. Infect. Dis. 64:389-395.

12. Choi JY, Ko EA, Kwon KT, Lee S, Kang CI, Chung $\mathrm{DR}$, et al. Acinetobacter sp. isolates from emergency departments in two hospitals of South Korea. J Med Microbiol. 2014;63:1363-8.

13. AA Alsultan, BA Evans, EA Elsayed, SI Al-Thawadi, AY Al-Taher, SGB Amyes, AM Al-Dughaym, A Hamou- 
da. High frequency of carbapenem-resistant Acinetobacter baumannii in patients with diabetes mellitus in Saudi Arabia. Journal of Medical Microbiology. (2013), 62, 885-888

14. Abeer M Baadani, , Sahar I Thawadi, , Noura A El-Khizzi, Ali S Omrani. Prevalence of colistin and tigecycline resistance in Acinetobacter baumanniiclinical isolates from 2 hospitals in Riyadh Region over a 2-year period. Saudi Med J. 2013; Vol. 34 (3):248-

15. CLSI, Clinical Laboratory Standard Institute. Methods for Dilution Antimicrobial Susceptibility Tests for Bacteria That Grow Aerobically; Approved Standard. Ninth ed; 2012. Document M07-A9. Wayne, PA

16. CLSI, Clinical Laboratory Standard Institute. PerformanceStandards for Antimicrobial Susceptibility Testing; Twenty-Six Informational Supplement. Document M100-S26,Wayne, PA; 2016.

17. European Committee on Antimicrobial susceptibility Testing.Breakpoint interpretation of MICs and zone diameters, Version 4.0; 2016.10.

18. Clinical and Laboratory Standards Institute. Performance standards for antimicrobial susceptibility testing; $24^{\text {th }}$ informational supplement; M100-S24. Wayne, PA: CLSI, 2014.

19. Pogue JM, Mann T, Barber KE, Kaye KS. Carbapenem-resistant Acinetobacter baumannii: epidemiology, surveillance and management. Expert Rev Anti Infect Ther. 2013; 11:383-93.

20. Voichița Lăzureanu, Mirela Poroșnicu, Ciprian Gândac2, Teodora Lăzureanu et al. Infection with Acinetobacter baumannii in an intensive care unit in the Western part of Romania. BMC Infectious Diseases. 2016, 16(Suppl 1):95

21. Moghadam MN, Motamedifar M, Sarvari J, Sedigh Ebrahim-Saraie H, Mousavi Same M, Moghadam FN. Emergence of Multidrug Resistance and Metallo-beta-lactamase Producing Acinetobacter baumannii Isolated from Patients in Shiraz, Iran. Annals of Medical and Health Sciences Research. | May-Jun 2016 | Vol 6 | Issue 3 |162167

22. Solomennyi A, Goncharov A, Zueva L. Extensively drug-resistant Acinetobacter baumannii belonging to theinternational clonal lineage $\mathrm{I}$ in a Russian burn intensive care unit. Int J Antimicrob Agents. 2015;45:525-8.

23. Teo J, LimTP, Hsu LY, Tan TY, Sasikala S, Hon PY, et al. Extensively drug-resistant Acinetobacter baumannii in a Thai hospital: a molecular epidemiologic analysis and identification of bactericidal Polymyxin B-based combi- nations. Antimicrob Resist Infect Control. 2015;4:2.

24. Azimi L, Talebi M, Pourshafie MR, Owlia P, RastegarLA. Characterization of carbapenemases in extensively drug resistance Acinetobacter baumannii in a burn care center in Iran. Int J Mol Cell Med. 2015;4:46-53.

25. Peleg AY, Seifert H, Paterson DL. Acinetobacter baumannii: emergence of a successful pathogen. Clin Microbiol Rev. 2008; 21:538- 82. PubMed

26. Munoz-Price LS, Arheart K, Nordmann P, et al. Eighteen years of experience with Acinetobacter baumannii in a tertiary care hospital. Crit Care Med. 2013; 41:273342. PubMed

27. Lesho E, Yoon EJ, McGann P, et al. Emergence of colistin-resistance in extremely drug-resistant Acinetobacter baumannii containing a novel pmrCAB operon during colistin therapy of wound infections. J Infect Dis. 2013; 208:1142-51. PubMed

28. Lopez-Rojas R, McConnell MJ, Jimenez-Mejias ME, Dominguez- Herrera J, Fernandez-Cuenca F, Pachon J. Colistin resistance in a clinical Acinetobacter baumannii strain appearing after colistin treatment: effect on virulence and bacterial fitness. AntimicrobAgents Chemother. 2013; 57:4587-9.

29. Pelletier MR, Casella LG, Jones JW, et al. Unique structural modifications are present in the lipopolysaccharide from colistin-resistant strains of Acinetobacter baumannii. Antimicrob Agents Chemother. 2013; 57:4831-40

30. O'Hara JA, Ambe LA, Casella LG, et al. Activities of vancomycin containing regimens against colistin-resistant Acinetobacter baumannii clinical strains. Antimicrob Agents Chemother 2013; 57:2103-8. PubMed

31. O Oikonomou, S Sarrou, CC Papagiannitsis, S Georgiadou, K Mantzarlis, E Zakynthinos, GN Dalekos, E Petinaki, Oikonomou et al. Rapid dissemination of colistin and carbapenem resistant Acinetobacter baumanniïn Central Greece: mechanisms of resistance, molecular identification and epidemiological data. BMC Infectious Diseases (2015). 15:559

32. Zubair A. Qureshi Lauren E. Hittle Jessica A. O'Hara Jesabel I. Rivera Alveena Syed Ryan K. Shields Anthony W. Pasculle Robert K. Ernst Yohei Doi. Colistin-Resistant Acinetobacter baumannii: Beyond Carbapenem Resistance: Clin Infect Dis. (2015) 60 (9): 1295-1303.

33. Gul R. Yilmaz,a Murat Dizbay,b Tumer Guven,c Husnu Pullukcu,d Meltem Tasbakan, d Ozlem Tunccan Guzel,bRisk factors for infection with colistin-resis- 
tant gram-negative microorganisms: a multicenter study. ASM; 36 | ISSUE 3 | MAY-JUNE 2016.

34. Leite GC, Oliveira MS, Perdigao-Neto LV, Rocha CK, Guimaraes T, et al. 2016) Antimicrobial Combinations against Pan-Resistant Acinetobacter baumannii Isolates with Different Resistance Mechanisms. PLoS One. 11:e0151270.
35. Mohan Ju, Dongni Hou, Shu Chen, Ying Wang, Xinjun Tang, Jie Liu , Cuicui Chen, Yuanlin Song, HuayinLi. Risk factors for mortality in ICU patients with Acinetobacter baumannii ventilator-associated pneumonia: impact of bacterial cytotoxicity. J Thorac Dis. 2018;10(5):26082617 